DOI:

10.1038/nrg2113

URLs

PRP2

http://www.ncbi.nlm.nih.gov/ entrez/query.fcgi?db=gene\&c $\mathrm{md}=$ Retrieve\&dopt=full report\&list_uids $=855745$

\title{
A not-so-passive spliceosome
}

The coding portions of eukaryotic genes are interrupted by introns, which must be efficiently and precisely removed from transcripts before translation. Intron removal is the job of the spliceosome - a large riboprotein machine. A recent report reveals a complex relationship between this complex and its substrates, raising the possibility that, instead of being a generic processor of transcripts, the spliceosome might be a specific regulator of gene expression.

Although introns vary in size and sequence, spliceosome function has been studied in only a small subset of genes. Pleiss et al. used a microarraybased assay that allowed them to distinguish between spliced and unspliced forms of intron-containing transcripts to look at intron removal on a genome-wide scale in budding yeast.

In the first set of experiments, the authors followed changes in splicing in strains that carried mutations in $P R P 2$, an essential component of the spliceosomal machinery. Although, as might be expected, unspliced precursor species accumulated in these mutants, it was clear that not all transcripts followed the same pattern. The authors then extended their analysis to 18 different mutations in core spliceosome components and 5 other mutations that affect mRNA processing. Although many of these mutations have effects on a broad range of transcripts, there are important, hitherto unanticipated differences. Splicing of some transcripts

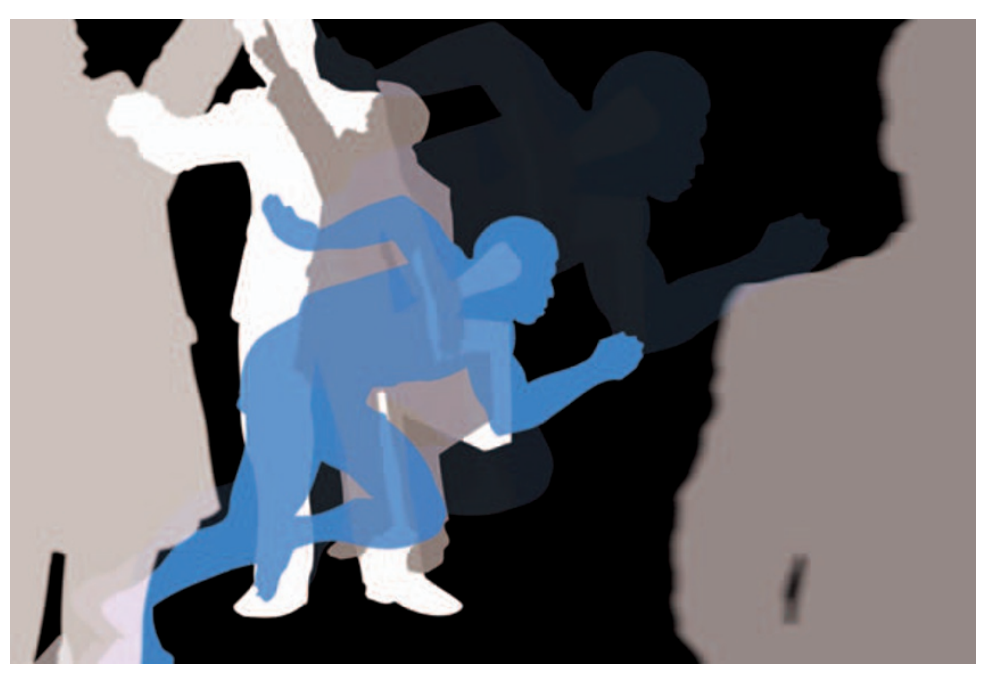

is equally affected by the different mutations, some transcripts are affected to different degrees by different mutations, and others are affected by only a subset of the mutations. Looking at the results from the point of view of the spliceosomal components, it seems that the requirement for a given spliceosomal component varies according to the identity of the RNA substrate.

Yeast intron-containing transcripts are relatively simple and similar to one another. Despite their efforts, the authors were unable to determine the mechanism by which the spliceosome is able to distinguish between transcripts. Detailed biochemical analysis will undoubtedly follow, as will examination of additional mutations. The high level of conservation of the mRNA splicing machinery implies that what Pleiss et al. see in yeast might also occur in other eukaryotes. In fact, it has been shown elsewhere that knocking down core spliceosomal components in the fly differentially affects alternative splicing.

\section{Magdalena Skipper}

ORIGINAL RESEARCH PAPER Pleiss, J. A. et al. Transcript specificity in yeast pre-mRNA splicing revealed by mutations in core spliceosomal components. PLoS Biol. 5, e90 (2007) FURTHER READING Xing, Y. \& Lee, C. Alternative splicing and RNA selection pressure - evolutionary consequences for eukaryotic genomes. Nature Rev. Genet. 7, 499-509 (2006)| Roy, S. W. \& Gilbert, W. The evolution of spliceosomal introns: patterns, puzzles and progress. Nature Rev. Genet. 7, 211-221 (2006) 